\title{
Coimbatore Orthopedic Surgical Fixation Protocol in COVID-19 Pandemic_-Our Experience
}

\author{
Vetrivel Chezian Sengodan ${ }^{1}$, Mugundhan Moongilpatti Sengodan², Ramachandran Perumal ${ }^{3}$
}

\begin{abstract}
Introduction: The severe acute respiratory syndrome coronavirus 2 (SARS-CoV-2) coronavirus disease-2019 (COVID-19) outbreak became a pandemic across the world after the outbreak in China in December 2019. This study aimed to determine the impact on the emergency orthopedic fixation during the COVID-19 pandemic in the Institute of Orthopaedics and Traumatology, Coimbatore.

Materials and methods: This is a retrospective study done on patients of trauma admitted in the emergency orthopaedic unit, under Tamilnadu Accident and Emergency Care Initiative (TAEI), Coimbatore Medical College Hospital from April 2020 to December 2020. All the patients admitted were evaluated as per the routine protocols followed in this COVID-19 pandemic in our institution and the patients were taken up for emergency orthopedic fixation based on the COVID-19 status.

Results: The total number of emergency orthopedic surgical fixation procedures done during the pandemic period from April 2020 to December 2020 was 245 . Orthopedic surgical fixation done in elective theatres during this pandemic in 2020 is 245 cases. As per the results, during the COVID-19 pandemic, emergency orthopedic surgical fixation was not stopped and treated as per the COVID-19 protocol followed in our hospital. Conclusion: The SARS-CoV-2 (COVID-19) pandemic has a major impact on orthopedic surgical fixation. All the patients admitted should be evaluated for the COVID-19 infection and use of adequate personal protective equipment while treating the patients to deliver safe and effective surgical services during this COVID-19 pandemic.
\end{abstract}

Keywords: Coimbatore, COVID-19 pandemic, Orthopedic, Protocol, Surgical fixation.

Journal of Orthopedics and Joint Surgery (2021): 10.5005/jp-journals-10079-1044

\section{INTRODUCTION}

The severe acute respiratory syndrome coronavirus 2 (SARS-CoV-2) causes the coronavirus disease-2019 (COVID-19). This disease spreads all over the world causing severe acute respiratory syndrome due to coronavirus 2. ${ }^{1}$ Coronavirus disease-2019 has made a big impact in all spheres of life. ${ }^{2}$ The World Health Organization (WHO) announced it as a pandemic on March 11, 2020. ${ }^{3}$ In India, the first case of COVID19 was reported in Kerala. ${ }^{4}$ Gradually it affected all over India. The Government of India (GOI) has announced the nationwide lockdown to break the chain of transmission of COVID-19. In the initial phase, due to lack of knowledge, and unpreparedness to deal with a large number of cases of this disease, a lot of confusion happened in the patient management and decision-making. ${ }^{6}$ In the early phase of the COVID-19 pandemic, there was no protocol for the healthcare professionals to be followed in orthopedic surgical fixation except utilizing the personal protective gadgets. In the early phase of the pandemic, private hospitals were reluctant to take up the case for orthopedic surgical fixation, hence the government tertiary care centers were the only centers to operate the injured persons. Healthcare professionals irrespective of their specialty were diverted to COVID designated wards in government tertiary care centers. We have analyzed the impact of the COVID-19 pandemic in orthopedic surgical fracture fixation in our institution, using Coimbatore orthopedic surgical fixation COVID-19 protocol.

\section{Materials and Methods}

The study was done in the Institute of Orthopaedics and Traumatology, Coimbatore Medical College Hospital, Coimbatore. The study period was from April 2020 to December 2020. All the orthopedic patients admitted in the emergency ward in our institution were included in the study and the trauma cases without
${ }^{1-3}$ Institute of Orthopaedics and Traumatology, Coimbatore Medical College and Hospital, Coimbatore, Tamil Nadu, India

Corresponding Author: Vetrivel Chezian Sengodan, Institute of Orthopaedics and Traumatology, Coimbatore Medical College and Hospital, Coimbatore, Tamil Nadu, India, Phone: +91 9843028096, e-mail: svcortho@gmail.com

How to cite this article: Sengodan VC, Sengodan MM, Perumal R. Coimbatore Orthopedic Surgical Fixation Protocol in COVID-19 Pandemic_-Our Experience. J Orth Joint Surg 2021;3(1):1-6.

Source of support: Nil

Conflict of interest: None

fracture were excluded in the study. We followed a unique protocol named the Coimbatore orthopedic surgical fixation protocol in the COVID-19 pandemic (Flowchart 1). According to our protocol at the entry-level, all the orthopedic patients admitted in the emergency room have to undergo throat and nasopharyngeal swab which will be sent for reverse transcription-polymerase chain reaction (RTPCR)/Cartridge Based Nucleic Acid Amplification Test (CBNAAT) assay for SARS-CoV-2 and CT chest for COVID-19 Reporting and Data System (CO-RADS) to rule out the involvement of lung due to COVID19 infection. Due to the limited laboratory personnel and limited resources, the blood investigations indicating the severity of the COVID-19 infection such as interleukin-6, D-dimer, and C-reactive protein were not done routinely.

As per our protocol, COVID-19 positive cases with compound fractures were operated in COVID-19 designated operation theater in our hospital and the negative cases with compound fractures were operated in the emergency orthopedic theater for orthopedic procedures. The operative personal will wear the personal

(c) The Author(s). 2021 Open Access This article is distributed under the terms of the Creative Commons Attribution 4.0 International License (https:// creativecommons.org/licenses/by-nc/4.0/), which permits unrestricted use, distribution, and non-commercial reproduction in any medium, provided you give appropriate credit to the original author(s) and the source, provide a link to the Creative Commons license, and indicate if changes were made. The Creative Commons Public Domain Dedication waiver (http://creativecommons.org/publicdomain/zero/1.0/) applies to the data made available in this article, unless otherwise stated. 
Flowchart 1: Coimbatore orthopedic surgical fixation COVID-19 protocol

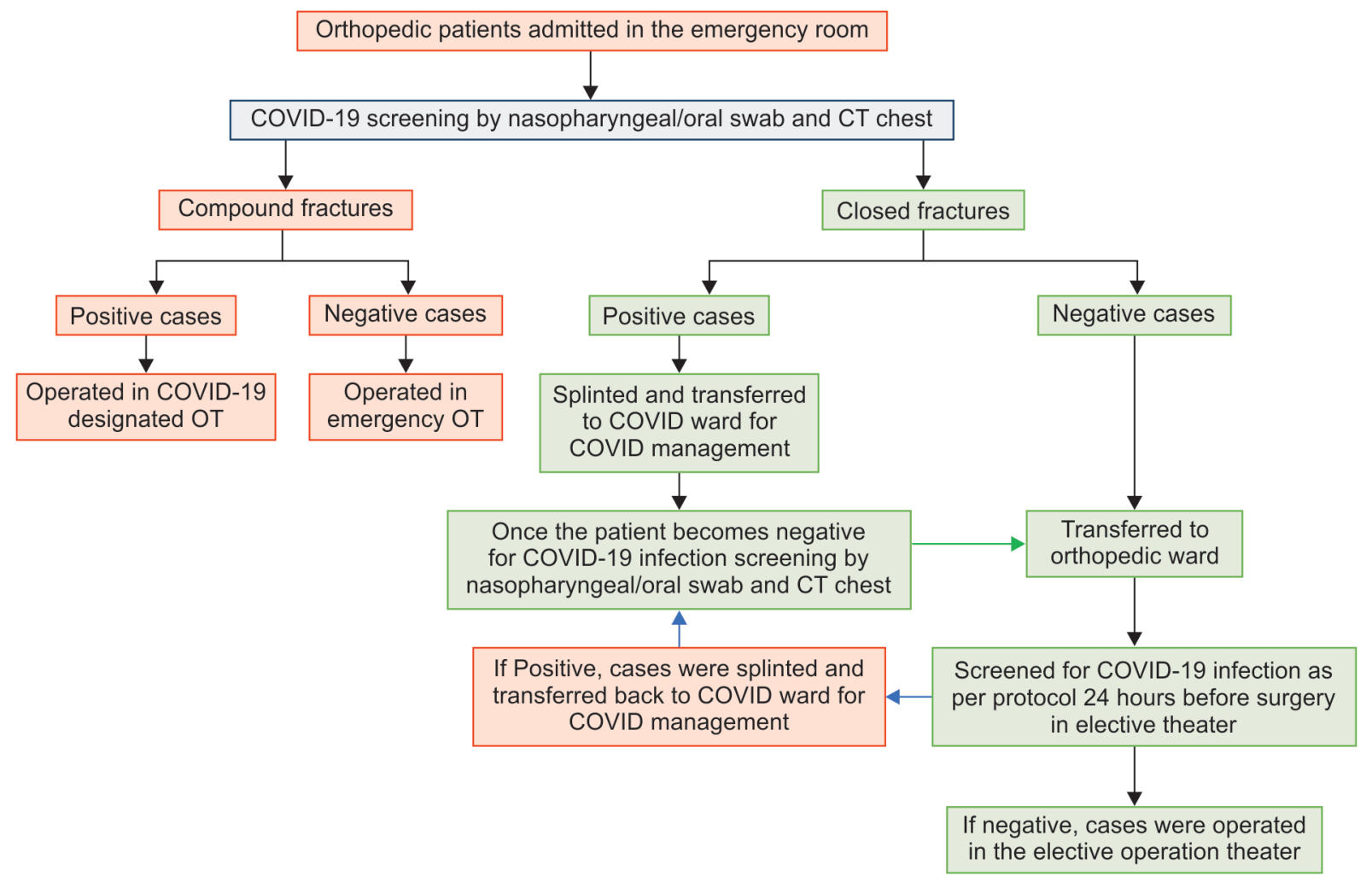

protective equipment available in the operation theater to prevent COVID-19 cross-infection. The following emergency orthopedic procedures like intramedullary nailing of long bones (IMIL nailing), external fixator application, plating, tension band wiring, cerclage wiring, limited internal fixation like $k$ wire fixation, and traumatic amputation were done (Figs 1 to 3 and Table 1).

Coronavirus disease-2019 positive cases with closed fractures were splinted and transferred to the COVID-19 ward in our hospital for COVID management. Once the patient becomes negative for COVID-19 infection, the patients were transferred back to the orthopedic ward. In orthopedic ward COVID-19, negative cases were again screened for COVID-19 infection 24 hours before surgery in elective theater by the throat and nasopharyngeal swab test for RTPCR/CBNAAT assay for SARS-CoV-2 and CT chest for CO-RADS. In our institution, we used to get the COVID-19 swab result within 3-6 hours and CT chest for CO-RADS within 1 hour.

Using the protocol, the transmission of the COVID-19 infection is reduced and the mortality of the fracture patients with COVID-19 is nil. The advantages of the Coimbatore orthopedic surgical fixation COVID-19 protocol are early identification of the COVID-19 positive cases among the orthopedic patients in the emergency room at the entry-level, early segregation of the COVID-19 positive cases from the negative cases, early orthopedic surgery within golden hours for a patient infected with COVID-19, early transfer of COVID-19 positive patients to COVID ward for COVID management to avoid late complications, preventing the contamination of the emergency theater, and reduce the chances of occupational transmission of COVID-19 among healthcare professionals in the emergency operation theater. Screening the orthopedic cases 24 hours before surgery in elective operation theater will reduce the spread of COVID-19 infection in elective operation theater and reduce the chances of occupational transmission of COVID-19 among healthcare workers and healthcare professionals. All the operating staff were instructed to wear personal protective gadgets (Fig. 4) in the emergency room and operation theater (COVID designated OT, emergency OT, elective OT) since power tools and electrocautery were used in the operation room which produces potentially infective aerosol which may lead to occupation transmission of COVID-19 by aerosolization of blood and other body fluids.

\section{Results}

The total number of emergency orthopedic surgical fixation procedures done during the pandemic period from April 2020 to December 2020 was 245 (Table 2 and Fig. 5). The orthopedic surgical fixation done in elective theaters during this pandemic in 2020 is 245 cases (Table 3 and Fig. 6).

As per our results, during the COVID-19 pandemic emergency orthopedic surgical fixation was not stopped in our hospital. According to our study in the emergency ward, 105 orthopedic patients were found to have COVID-19 infection. Nasopharyngeal and throat swab alone was positive among 41 patients, CT chest alone was positive among 29 patients, and both swab and CT chest were positive among 35 patients.

In December 2018, before the COVID-19 pandemic, 47 patients were operated on for emergency orthopedic surgery whereas, during the COVID-19 pandemic in December 2020, 48 patients were operated on. In November 2019, before the COVID-19 pandemic 37 patients were taken up for emergency orthopedic surgical fixation whereas, during the COVID-19 pandemic in November 2020, 45 patients were operated on for emergency orthopedic surgical 

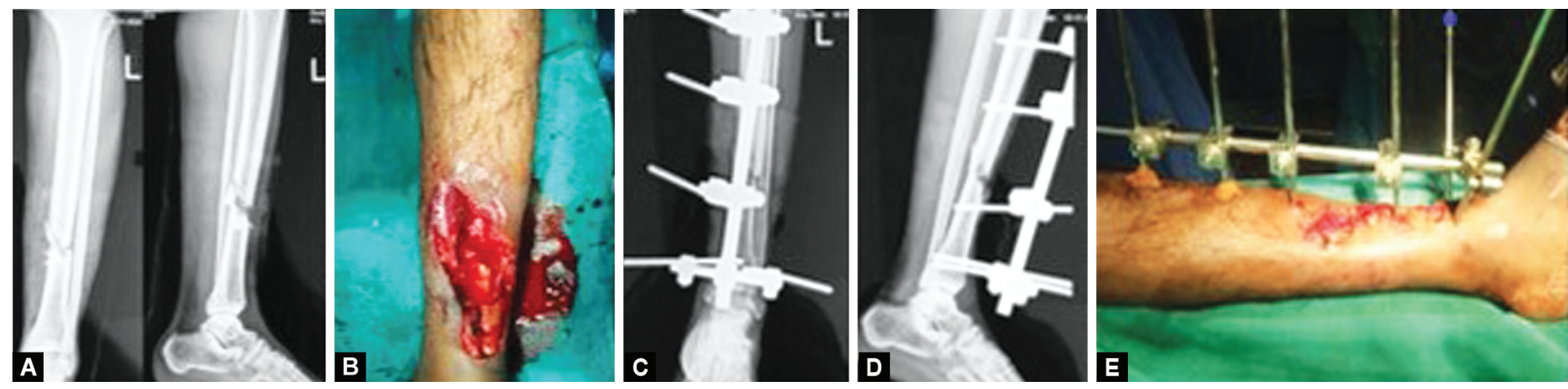

Figs $1 \mathrm{~A}$ to $\mathrm{E}$ : External fixation of compound tibial fracture
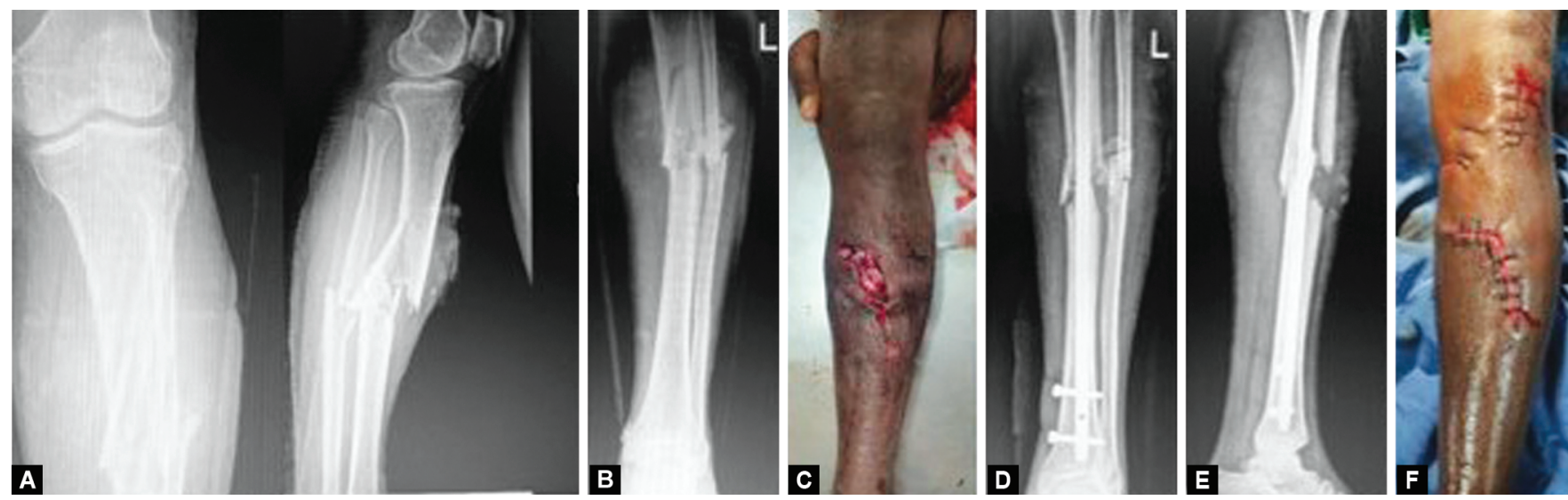

Figs $2 A$ to $F$ : Intramedullary nailing of compound tibial fracture

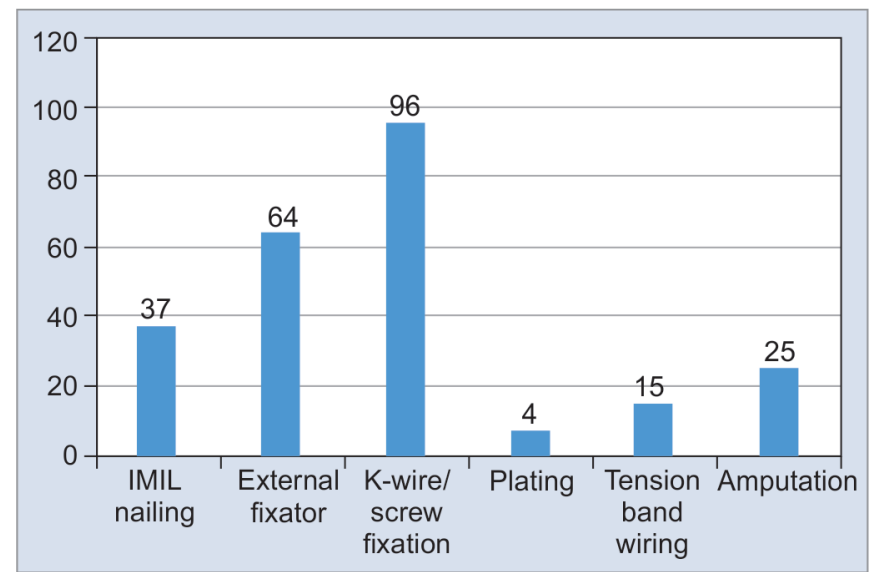

Fig. 3: Emergency major surgical fixations done during the pandemic period

fixation (Table 2 and Fig. 5). The total number of compound fractures that underwent external fixation in the COVID-19 operation theater in December 2020 was more than the external fixation in the year 2019 (Table 4 and Figs 7 and 8). The increased number of major surgery during the COVID-19 pandemic may be due to the lockdown of operation theaters in private hospitals.

In our study, we have not observed any local or systemic complication observed in COVID-19 positive patients operated in the emergency theater as well as elective theater. In our study, blood transfusion was done in 26 patients. In our study, superficial

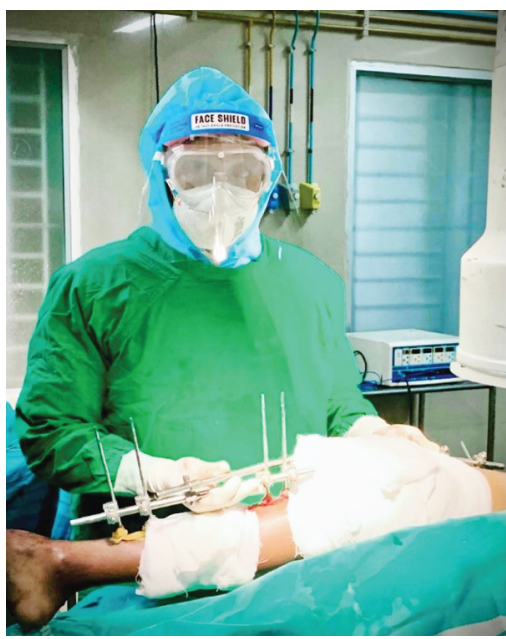

Fig. 4: Surgeon with personal protective gadgets

Table 1: Emergency major surgical fixation done during the pandemic period

\begin{tabular}{llc}
\hline S. no. & Major cases & 2020 \\
\hline 1 & Total no of IMIL nailing & 37 \\
2 & Total no of external fixator & 64 \\
3 & Total no of $K$ wire/screw fixation & 96 \\
4 & Total no of plating for long bones & 8 \\
5 & Total no of tension band wiring & 15 \\
6 & Total no of amputations & 25 \\
& Total & 245 \\
\hline
\end{tabular}


Table 2: Orthopedic surgeries done in emergency theaters before and during the COVID-19 pandemic

\begin{tabular}{|c|c|c|c|c|c|c|}
\hline \multirow[b]{2}{*}{ Month } & Major & Minor & Major & Minor & Major & Minor \\
\hline & \multicolumn{2}{|c|}{2018} & \multicolumn{2}{|c|}{2019} & \multicolumn{2}{|c|}{2020} \\
\hline April & 58 & 12 & 59 & 40 & 9 & 54 \\
\hline May & 58 & 10 & 56 & 33 & 23 & 119 \\
\hline June & 47 & 15 & 78 & 20 & 27 & 95 \\
\hline July & 60 & 30 & 47 & 36 & 12 & 68 \\
\hline August & 58 & 50 & 70 & 43 & 24 & 180 \\
\hline September & 58 & 54 & 32 & 46 & 25 & 113 \\
\hline October & 60 & 49 & 63 & 47 & 32 & 125 \\
\hline November & 70 & 42 & 37 & 30 & 45 & 167 \\
\hline December & 47 & 39 & 49 & 17 & 48 & 148 \\
\hline Total & 516 & 301 & 491 & 312 & 245 & 1,069 \\
\hline
\end{tabular}

Table 3: Orthopedic surgeries done in elective theaters before and during the COVID-19 pandemic

\begin{tabular}{|c|c|c|c|c|c|c|c|c|c|}
\hline \multirow[b]{2}{*}{ Month } & Major cases & Minor cases & Total cases & Major cases & Minor cases & Total cases & Major cases & Minor cases & Total cases \\
\hline & \multicolumn{3}{|c|}{2018} & \multicolumn{3}{|c|}{2019} & \multicolumn{3}{|c|}{2020} \\
\hline April & 128 & 395 & 523 & 182 & 418 & 600 & 0 & 33 & 33 \\
\hline May & 143 & 403 & 546 & 173 & 383 & 556 & 13 & 100 & 113 \\
\hline June & 162 & 395 & 557 & 112 & 222 & 334 & 22 & 127 & 149 \\
\hline July & 196 & 138 & 334 & 150 & 461 & 611 & 15 & 167 & 168 \\
\hline August & 181 & 458 & 639 & 169 & 512 & 781 & 10 & 164 & 174 \\
\hline September & 168 & 319 & 487 & 146 & 344 & 490 & 26 & 149 & 175 \\
\hline October & 162 & 312 & 474 & 169 & 470 & 639 & 38 & 219 & 257 \\
\hline November & 156 & 321 & 477 & 172 & 390 & 562 & 45 & 257 & 302 \\
\hline December & 184 & 312 & 496 & 173 & 383 & 556 & 76 & 208 & 284 \\
\hline Total & 1,480 & 3,130 & 4,610 & 1,446 & 3,583 & 5,129 & 245 & 1,424 & 1,645 \\
\hline
\end{tabular}

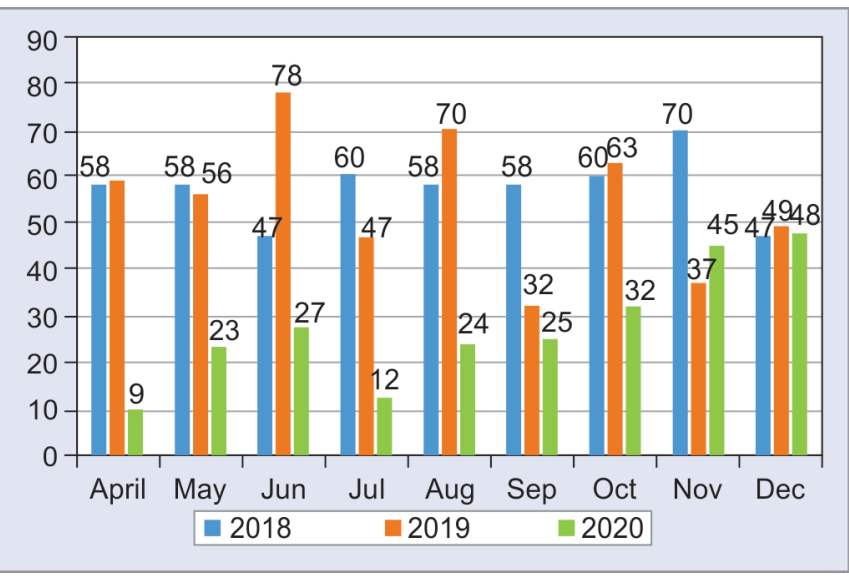

Fig. 5: Orthopedic surgeries done in emergency theaters before and during the COVID-19 pandemic

wound infection was present among eight patients and was treated with systemic antibiotics.

\section{Discussion}

Coronavirus disease-2019 is a viral disease. Coronavirus disease2019 is also known as SARS-CoV-2. Coronavirus is an RNA virus. The entire world is affected by COVID-19 causing a global pandemic. ${ }^{7}$ The lockdown was enforced all over the world. Healthcare workers

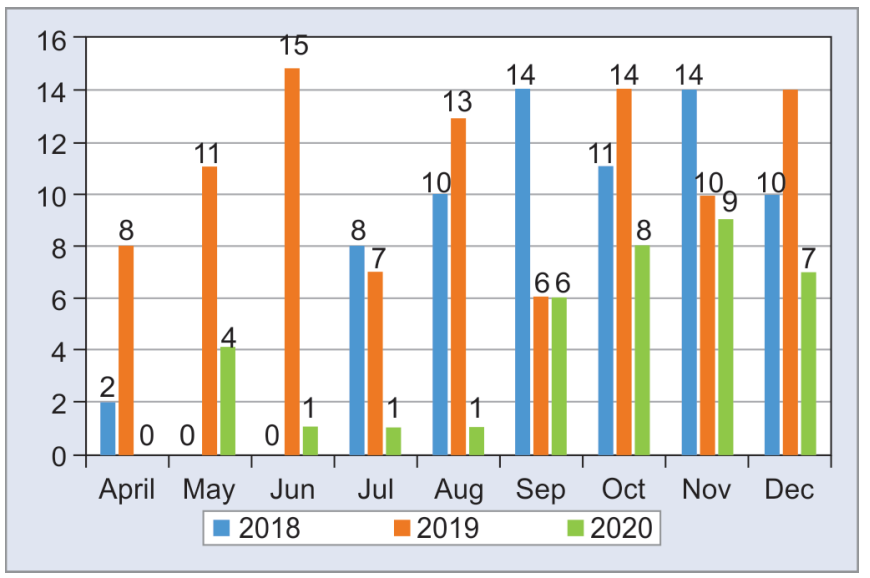

Fig. 6: Orthopedic surgeries done in elective theaters before and during the COVID-19 pandemic

all over the world have worked at their level best during the COVID19 pandemic.

During the COVID-19 pandemic, there was a reduction in orthopedic surgical fixation all over the world. As per the study in Italy by Giuntoli et al., the trauma including orthopedic fracture during the COVID-19 pandemic was reduced. ${ }^{8}$ In India, according to Dhillon et al., there was a significant decrease in the trauma cases in Chandigarh during the lockdown period. ${ }^{9}$ Battiato et al. have also observed a significant reduction in elective surgery in 
Table 4: External fixation and IMIL nailing done in emergency theaters before pandemic 2018-2019 and during the COVID-19 pandemic 2020

\begin{tabular}{|c|c|c|c|c|c|c|}
\hline \multirow[b]{2}{*}{ Month } & IMIL nailing & External fixator & IMIL nailing & External fixator & IMIL nailing & External fixator \\
\hline & \multicolumn{2}{|c|}{2018} & \multicolumn{2}{|c|}{2019} & \multicolumn{2}{|c|}{2020} \\
\hline April & 2 & 15 & 8 & 16 & 0 & 2 \\
\hline May & 0 & 17 & 11 & 18 & 4 & 7 \\
\hline June & 0 & 13 & 15 & 28 & 1 & 12 \\
\hline July & 8 & 9 & 7 & 9 & 1 & 3 \\
\hline August & 10 & 13 & 13 & 20 & 1 & 2 \\
\hline September & 14 & 18 & 6 & 15 & 6 & 8 \\
\hline October & 11 & 14 & 14 & 15 & 8 & 4 \\
\hline November & 14 & 18 & 10 & 12 & 9 & 13 \\
\hline December & 10 & 12 & 14 & 11 & 7 & 13 \\
\hline Total & 69 & 129 & 98 & 144 & 37 & 64 \\
\hline
\end{tabular}

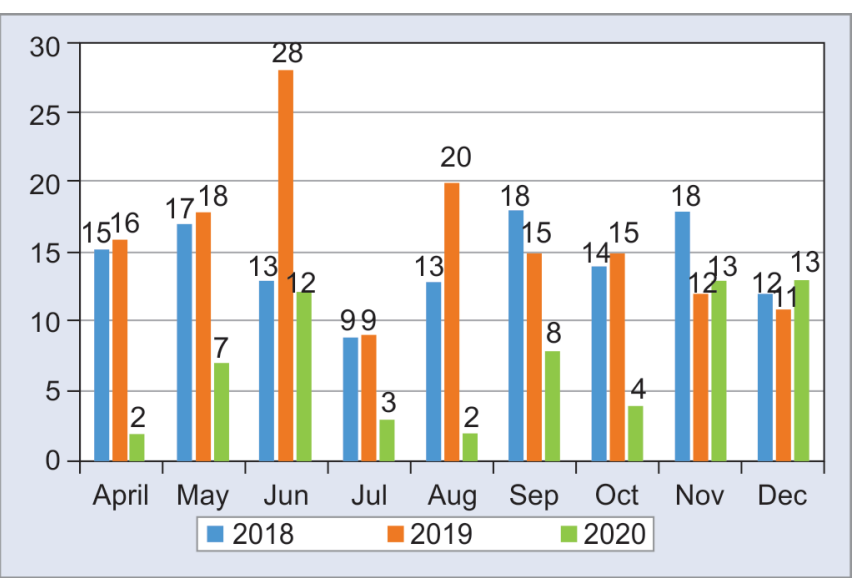

Fig. 7: Intramedullary nailing done in emergency theaters before and during the COVID-19 pandemic

central Italy. ${ }^{10}$ In the USA, Bedard et al. have observed a significant reduction in arthroplasty volume. ${ }^{11}$ Best et al. have observed a significant reduction of elective orthopedics throughout the United States of America. ${ }^{12}$ In India, according to Kumar Jain et al., the reduction of orthopedic cases was due to (a) strict lockdown made the people stay in the home, (b) reduced road traffic accident due to decreased transport, (c) hospitals planning to save the already depleted resources, (d) to minimize the spread of COVID-19 infection among healthcare professionals, and (e) decline in the sporting and industrial activities. ${ }^{13}$ Wallace et al. from the United Kingdom have observed an unprecedented impact on all aspects of trauma and orthopedic services due to the COVID-19 pandemic. ${ }^{14}$ Wong and Cheung from Hong Kong observed a significant reduction in orthopedic operations. ${ }^{15}$ On reviewing the global burden of trauma, there was a worldwide reduction in the footfalls of trauma in Asia, Australia, Europe, the United States of America, the United Kingdom, and New Zealand. ${ }^{16}$

During this COVID-19 pandemic, in the initial phase, there was a reduction of the number of orthopedic surgical fixation in our institute due to strict lockdown. Once the government announces the relaxation of lockdown needy people with trauma and other painful musculoskeletal problems started to visit the hospitals seeking treatment. ${ }^{17}$

In the acute phases of the COVID-19 pandemic, the American College of Surgeons (ACS) has issued the following guidelines

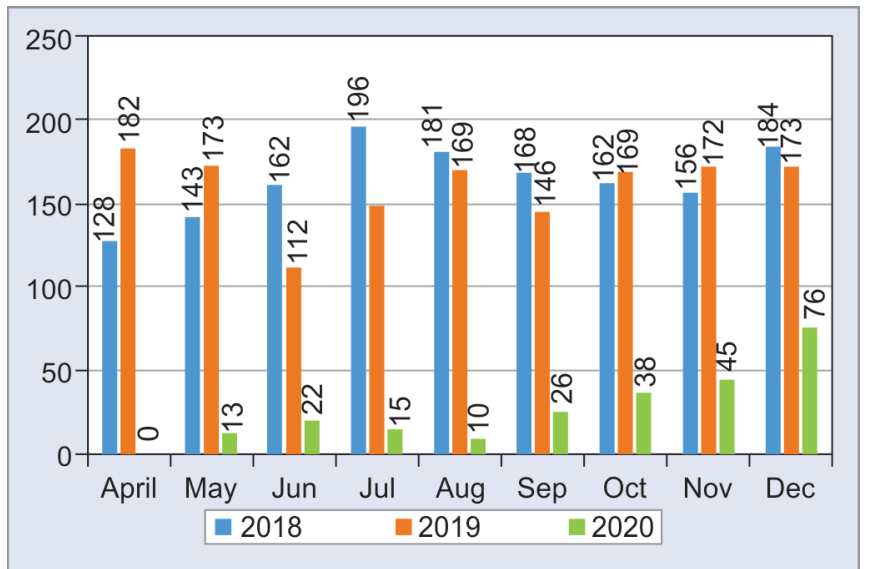

Fig. 8: External fixation done in emergency theaters before and during the COVID-19 pandemic

regarding surgery. Phase 1 is called the preparation phase where few COVID-19 patients with hospital resources not exhausted and surgery should be restricted to the patients whose survival endangered within 3 months of injury, Phase 2 is called the escalation phase where many COVID-19 patients with limited hospital resources and the surgery is restricted to the patients whose survival endangered within few days of injury and in phase 3 where hospital resources are all directed to COVID-19 patients-lack of ICU and ventilator capacity and surgery is done only for patients with life-threatening injuries whose survivorship compromised within next few hours. ${ }^{18}$ Based on the observation of Fineberg, patients must be treated according to their COVID19 exposure. He classifies the patients into five types (a) patients not exposed or infected, (b) patients exposed but asymptomatic, (c) patients recovered from the infection, (d) patients possibly infected but present with sign and symptoms (tested negative), (e) infected patient tested positive, and (f) sixth type was added into the account, patients with comorbidities. According to Fineberg, priority for surgery was given to the patients who have no signs of COVID-19 or recovered from the disease (type $a, b$, and c). Patients with COVID-19 infection suspected or confirmed (type d, e, and f) should have fully recovered and had an adequate immune response before considering surgery. ${ }^{19}$

The American Society of Anesthesiologists (ASA) has classified the patient operative outcome into various categories. Normal 
healthy individuals are under ASA I category. Patients with mild systemic disease like controlled systemic hypertension and type 2 diabetes are under the ASA II category. Patients with severe systemic disease that is not life-threatening like stable angina, morbid obesity, and uncontrolled diabetes are under the ASA III category. Patients with a severe systemic disease with a constant threat to life like myocardial infarction, cardiac failure, and sepsis are under the ASA IV category. ${ }^{20}$ In ASA I and II category, the asymptomatic patients were operated on. In ASA I and II with COVID-19 infected patients, the surgery was deferred. In patients under ASA III and ASA IV category, the surgery was deferred, even if they were COVID-19 negative with symptoms. In our hospital, we followed Coimbatore orthopedic surgical fixation COVID-19 protocol.

Our protocol is useful not only in early identification and segregation of the infected patients but also in early surgical fixation of deserving cases in the emergency operation theater and in avoiding contamination and cross-infection in the elective theater. In Tamil Nadu, Tamilnadu Accident Emergency Care Initiative services were provided without disruption even during the COVID-19 pandemic. We also want to enlighten the orthopedic trauma services that were provided as usual in our institution as per our protocol.

\section{Conclusion}

Coronavirus disease-2019 has imposed a massive burden on health systems around the world. In our institution, we worked our level best to provide safe and effective orthopedic surgical treatment to fractured patients during the COVID pandemic period. We followed Coimbatore orthopedic surgical fixation COVID-19 protocol. A large multicenter study may be necessary to confirm the utility of Coimbatore orthopedic surgical fixation COVID-19.

\section{References}

1. Zhou F, Yu T, Du R, et al. Clinical course and risk factors for mortality of adult inpatients with COVID-19 in Wuhan, China: a retrospective cohort study. Lancet 2020;395(10229):1054-1062. DOI: 10.1016/S01406736(20)30566-3.

2. Haleem A, Javaid M, Vaishya R, et al. Effects of COVID-19 pandemic in the field of orthopaedics. J Clin Orthop Trauma 2020;11(3):498-499. DOI: 10.1016/j.jcot.2020.03.015.

3. WHO Director-General's opening remarks at the media briefing on COVID19-March 2020 [Google Scholar].

4. Andrews MA, Areekal B, Rajesh KR, et al. First confirmed case of COVID-19 infection in India: a case report. Indian J Med Res 2020;151(5):490-492. DOI: 10.4103/ijmr.IJMR_2131_20.

5. COVID-19 lockdown in India. Wikipedia (Last accessed on 15th January 2021). https://en.wikipedia.org/wiki/COVID-19_lockdown_in_ India.
6. Askari $A$, Arasteh $P$, Jabalameli $M$, et al. COVID-19 and orthopaedic surgery: experiences from Iran.J Bone Joint Surg Am 2020;102(13):11261128. DOI: 10.2106/JBJS. 20.00631.

7. Jogalekar MP, Veerabathini A, Gangadaran P. Novel 2019 coronavirus: genome structure, clinical trials, and outstanding questions. Exp Biol Med (Maywood) 2020;245(11):964-969. DOI: 10.1177/1535370220920540.

8. Giuntoli M, Bonicoli E, Bugelli G, et al. Lessons learnt from COVID 19: an Italian multicentric epidemiological study of orthopaedic and trauma services. J Clin Orthop Trauma 2020;11(4):721-727. DOI: 10.1016/j.jcot.2020.05.021.

9. Dhillon MS, Kumar D, Saini UC, et al. Changing pattern of orthopaedic trauma admissions during COVID-19 pandemic: experience at a tertiary trauma centre in India. Indian J Orthop 2020;54(Suppl 2):1-6. DOI: 10.1007/s43465-020-00241-0.

10. Battiato C, Berdini M, Luciani P, et al. Impact of coronavirus disease 2019 (COVID-19) on the epidemiology of orthopedics trauma in a region of central Italy. Injury 2020;51(12):2988-2989. DOI: 10.1016/j. injury.2020.09.044.

11. Bedard NA, Elkins JM, Brown TS. Effect of COVID-19 on hip and knee arthroplasty surgical volume in the United States. J Arthroplast 2020;35(7S):S45-S48. DOI: 10.1016/j.arth.2020.04.060.

12. Best MJ, Aziz KT, McFarland EG, et al. Economic implications of decreased elective orthopaedic and musculoskeletal surgery volume during the coronavirus disease 2019 pandemic. Int Orthop 2020;44(11):2221-2228. DOI: 10.1007/s00264-020-04713-8.

13. Kumar Jain V, Lal H, Kumar Patralekh $\mathrm{M}$, et al. Fracture management during COVID-19 pandemic: a systematic review. J Clin Orthop Trauma 2020;11(Suppl 4):S431-S441. DOI: 10.1016/j.jcot.2020.06.035.

14. Wallace CN, Kontoghiorghe C, Kayani B, et al. The impact of COVID-19 on trauma and orthopedic surgery in the United Kingdom. Bone Jt Open 2020;1(7):420-423. DOI: 10.1302/2633-1462.17.

15. Wong JSH, Cheung KMC. Impact of COVID-19 on orthopaedic and trauma service: an epidemiological study. J Bone Joint Surg Am 2020;102(14):e80. DOI: 10.2106/JBJS.20.00775.

16. Waseem $S$, Nayar $S K$, Hull $P$, et al. The global burden of trauma during the COVID-19 pandemic: a scoping review. J Clin Orthop Trauma 2020;12(1):200-207. DOI: 10.1016/j.jcot.2020.11.005.

17. Vaishya R, Vaish A, Kumar A. Impact of COVID-19 on the practice of orthopaedics and trauma-an epidemiological study of the full pandemic year of a tertiary care centre of New Delhi. Int Orthop 2021;45(6):1391-1397. DOI: 10.1007/s00264-021-05021-5.

18. ACS. 2020. Guidelines for Triage and Management of Elective Cancer Surgery Cases during the Acute and Recovery Phases of Coronavirus Disease 2019 (COVID-19) Pandemic. https://www.facs.org//media/ files/covid19/acs_triage_and_management_elective_cancer_ surgery_during_acute_and_recovery_phases.ashx accessed 25 April 2020.

19. Fineberg HV. Ten weeks to crush the curve. N Engl J Med 2020;382(17):e37. DOI: 10.1056/NEJMe2007263.

20. Doyle DJ, Goyal A, Bansal P, et al. American Society of Anesthesiologists Classification. [Updated 2020 Jul 4]. In: StatPearls [Internet]. Treasure Island (FL): StatPearls Publishing; 2021. p. 2. 